\title{
miR-24 and its Role as an Oncogene in Cancer
}

\begin{abstract}
MicroRNAs (miRNAs) regulate the expression of genes involved in numerous cellular processes, including proliferation, metabolism differentiation and apoptosis. Changes in the expression levels of miRNAs are associated with the development of various diseases, including cancer. Dysregulation of miRNA expression has been demonstrated to have a role in tumorigenesis and it has been revealed that some miRNAs can act either as oncogenes or tumor suppressors in cancer. miRNA-24 has been identified as an oncogene in various types of cancer. This review discusses the function of miRNA-24 as oncogene in gastric cancer, breast cancer, pancreatic cancer, lung cancer and leukemia.
\end{abstract}

Keywords: microRNA-24; Oncogene; Cancer

\section{Abbreviations}

miRNAs: MicroRNAs; DFS: Disease-Free Survival; RFS: Recurrence-Free Survival; SOCS6: Suppressor of Cytokine Signaling 6; BCL2L11 or Bim: BCL2 like 11; EBC: Early Breast Cancer; ING: Inhibitor of Growth; NAIF1: Nuclear Apoptosis Inducing Factor 1; SOX7: Sex-Determining Region Y-box 7

\section{Background}

MicroRNAs (miRNAs) are small non-coding RNAs that negatively regulate gene expression by controlling translation or stability of mRNAs via an RNA interference-like pathway. miRNAs comprise $1-3 \%$ of the human genome and regulate the expression of $30 \%$ of human genes [1]. miRNA sequences can be located within the introns of protein-coding genes or in units that do not encode proteins, and are expressed coordinately. A few miRNA genes are located in gene exons.

One of the main functions of miRNAs is the silencing of gene expression, which occurs through binding of an miRNA to complementary sequences in the 3' Untranslated Region (3'UTR) of target mRNAs [2,3]. Many studies have demonstrated that miRNAs are associated with various cellular functions and important biological processes, such as development, hematopoiesis, aging and certain endocrine pathways. These small molecules also function as important regulators in the progression of many human diseases, including heart disease and cancer [4,5]. miRNAs can have oncogenic or anti-cancer effects by targeting tumor suppressor genes or oncogenes, respectively, whereby dysregulation and dysfunction of the gene expression can lead to the development and proliferation of cancer cells [4]. Recent research indicates that miRNAs have important roles in various aspects of cancer biology, including cell proliferation, angiogenesis, apoptosis, invasion, metastasis and drug resistance [4], miRNA (miR)-24 is one of the best known cancer-associated miRNAs and it is considered to be an oncomiR. The overexpression of miR-24 has been reported in various types of human cancer, including breast cancer [6], pancreatic cancer, lung cancer [7], gastric cancer and leukemia $[8,9]$.

\section{Journal of}

Human Anatomy \& Physiology

\section{Rivas-Alarcón A, Organista-Nava J, Illades-Aguiar $B$, Leyva-Vázquez MA* and Gómez-Gómez $\mathbf{Y}^{\star}$}

Laboratorio de Biomedicina Molecular, Facultad de Ciencias Químico-Biologicas, Universidad Autonoma de Guerrero, Chilpancingo, Guerrero, Mexico

\section{*Address for Correspondence}

Gomez-Gomez Y, Laboratorio de Biomedicina Molecular, Facultad de Ciencias Quimico-Biologicas, Universidad Autonoma de Guerrero, Chilpancingo, Guerrero, Mexico; E-mail: yazmigomezgomez@gmail.com Leyva-Vazquez MA, Laboratorio de Biomedicina Molecular, Facultad de Ciencias Químico-Biologicas, Universidad Autonoma de Guerrero, Chilpancingo, Guerrero, Mexico E-mail: leyvamarco13@gmail.com

Submission: 01 March, 2019

Accepted: 29 July, 2019

Published: 01 August, 2019

Copyright: ๑ 2019 ๑ 2019 Rivas-Alarcón A, et al. This is an open access article distributed under the Creative Commons Attribution License, which permits unrestricted use, distribution, and reproduction in any medium, provided the original work is properly cited.

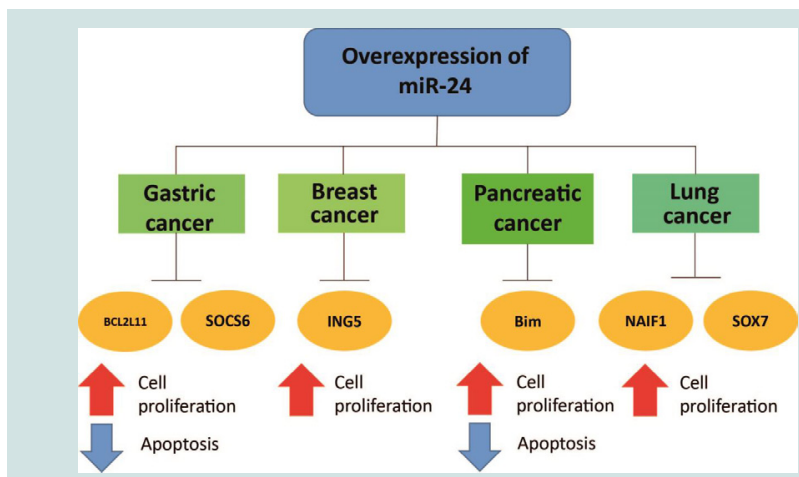

Figure 1: Expression of miR-24 and certain target genes in different types of cancer. Overexpression of miR-24 leads to a decrease in the expression of genes such as BCL2L11, SOCS6, ING5, NAIF1 and SOX7 that participate in important processes, including cell proliferation and apoptosis in different types of cancer. miR-24, microRNA-24; BCL2L11, BCL2 like 11; SOCS6, suppressor of cytokine signaling 6; ING5, inhibitor of growth 5; NAIF1, nuclear apoptosis inducing factor 1; SOX7, sex-determining region Y-box 7 .

Data published in a meta-analysis from 2018 suggested that alteration in the expression levels of miR-24 in various types of cancer may predict shorter survival [Hazard ratio, 2.49; 95\% confidence interval, $1.84-3.37 ; \mathrm{P}=0.0002$ ]. This association was predominantly observed in cancers of the digestive system, in which high expression of miR-24 indicated poor prognosis. This study also analyzed the association of tumor progression, Disease-Free Survival (DFS) and Recurrence-Free Survival (RFS) with the levels of miR-24 expression. This meta-analysis suggested that high expression of miR-24 may be associated with poor DFS, RFS and tumor progression [10].

The aim of this review is to know the role of miR-24 as an oncogene in different types of cancer, For this purpose, a search was made for articles indexed from 2015 to the date in which information 
was provided about miR-24 and its role as an oncogene in the carcinogenic process.

We can observe some target genes of miR-24 in different types of cancer (Figure 1). The overexpression of miR-24 leads to decrease in the expression of genes that involves in important processes in the development of carcinogenesis such as cell proliferation and apoptosis. In the following lines we will talk about some of them.

\section{miR-24 in Gastric Cancer}

The miR-23a/27a/24-2 cluster is made up of three miRNAs that are considered to be oncogenes in gastric cancer. It has been proposed that this cluster of miRNAs controls the cell cycle, proliferation, differentiation and cell death [11]. miR-23a and miR-24-2 have been reported to be overexpressed in gastric cancer tissue samples. The degree of alteration among miR-23a, miR-24 and miR-27 is variable; however, the overexpression of these miRNAs is consistent [11].

Transfection of gastric cancer cells with miR-23a, miR-24 and miR-27a inhibitors resulted in decreased cell growth. These observations indicate that miR-23a, miR-24 and miR-27a induce tumor development by promoting cell growth [11]. It has been proposed that miR-23a and miR-24 may regulate the progression of gastric cancer by reducing the expression of the target gene, suppressor of cytokine signaling 6 (SOCS6). A decrease in the expression of SOCS6 increases the activation of signals that promote cell proliferation and inhibit apoptosis, which may be associated with the reduced survival of patients with gastric cancer [11]. miR-23a, miR-24 and miR-27 could be useful targets for the development of alternative gastric cancer therapies [11].

In vivo and in vitro assays have demonstrated that miR-24 directly targets BCL2 like 11 (BCL2L11; also known as Bim), which is an important regulator of apoptosis. Zhang et al. analyzed the expression of miR-24 and BCL2L11 in gastric cancer tissue samples [8], noting that miR-24 was overexpressed and expression of BCL2L11 was decreased, resulting in increased cell proliferation and migration, and reduced apoptosis. In vivo studies have shown that overexpression of miR-24 accelerates tumor growth, while growth is inhibited by BCL2L11 overexpression. miR-24 and BCL2L11 may be important targets for future clinical research in gastric cancer [8].

The aforementioned studies suggest that miR-24 has targets genes involved in important processes associated with the development of gastric cancer (cell proliferation and apoptosis). Two of the genes that may be involved are SOCS6 and BCL2L11. miR-24 could be considered a therapeutic target in gastric cancer, as suppressing the expression or action of miR-24 would result in increased expression of its targets genes, potentially leading to reduced cell proliferation and enhanced apoptosis. These events could improve the prognosis in patients with gastric cancer.

\section{miR-24 and Breast Cancer}

miRNAs have an important role in the progression of breast cancer and could serve as useful cancer biomarkers. NanoString technology was used to analyze the expression of 800 miRNAs in plasma samples from patients with primary and metastatic breast cancer, with 29 miRNAs identified to be differently expressed in patients that developed metastasis compared with non-metastatic patients. Of these miRNAs, 24 were overexpressed and 5 were down regulated. miR-24 was overexpressed in the plasma and breast cancer tissue of patients with metastases compared with non-metastatic patients [12]. This overexpression as associated with advanced stage cancer and the triple negative/basal. Patients that exhibited overexpression of miR24 in primary tumors had a significantly lower survival rate compared to patients with low expression of miR-24 [12]; thus, miR-24 may be a involved in the development of metastasis in patients with breast cancer. This study also analyzed the gene expression in samples low and high miR-24 expression; in the samples with high expression of miR-24, 2,128 genes were upregulated and 1,190 genes downregulated compared with the low miR-24 samples. The differential analysis of gene expression showed that the main deregulated genes had functions in pathways associated with cancer and metastasis, such as survival, migration and proliferation [12]. miR-24 has been linked to the progression of breast cancer in several studies, and has been shown to regulate genes that control DNA damage repair and cell cycle progression [6,12-14].

One of the most important goals in breast cancer research is to identify patients that present with 'Early Breast Cancer (EBC)' as soon as possible and to determine which patients have a higher risk of relapse [13]. In a study by Basova et al. [13], four oncomiRs (miR-24, miR-19a, miR-155 and miR-181) and one tumor suppressor miR (let-7a) were identified in patients with EBC. The expression of these miRNAs was different between EBC patients with high and low relapse rates. Measuring the expression of miR-24, miR-19a, miR-155 and miR-181, and let-7a in patients with EBC could help to establish whether adjuvant therapy is required [13]. miR-155 and miR-24 were measured in serum from patients with Ki-67-positive tumors; these measurements were able to allows predicting the risk of relapse and could be used to help determine the most appropriate therapy for patients with EBC in the future [13]. Furthermore, combining the measurements of oncomiRs levels with the expression of Ki-67 could determine the risk of relapse in patients with EBC [13].

EBC refers to breast cancer that has not yet spread to axillary lymph nodes. Certain factors, including hormone receptors, and markers such as Her2 and Ki67, can help predict the risk of relapse in patients with $\mathrm{EBC}$; however, these methods are imprecise. The search for new biomarkers has indicated that miRNAs could help determine the risk of relapse with more precision. One of the miRNAs that could be useful is miR-24, as expression is low when a tumor is removed; therefore, its re-expression is associated with relapse in patients with breast cancer. Measuring the levels of miR-24 and other miRNAs, such as miR-155, may be independent risk factors that when used together with standardized risk factors might help identify patients with breast cancer that have a high risk of relapse and metastasis development.

The Inhibitor of Growth (ING) genes are a family of tumor suppressor's genes that are involved in several cellular processes, such as apoptosis, cell cycle regulation and chromatin remodeling [15]. ING5 is a member of the ING family with unknown function and regulation; however, downregulation of ING5 expression has been commonly observed in different cancer types [16]. It has been proposed that the expression of ING5 is decreased in breast cancer tissue samples [6]. Cui et al. determined the expression of ING5 after 
overexpressing and inhibiting miR-24 in breast cancer cell lines. Cell proliferation was increased and apoptosis was decreased in MCF-7 cells transfected with miR-24 mimics. The opposite effect occurred in transfected MCF-7 cells with miR-24 inhibitors. These results suggest that miR-24 decreases the expression of ING5, leading to an increase in cell proliferation and decrease in apoptosis in cells with breast cancer cells; thus, miR-24 and ING5 have opposing effects on cell proliferation, invasion and apoptosis in breast cancer [6].

\section{miR-24 and Pancreatic Cancer}

Pancreatic cancer has a high incidence worldwide, accounting for $\sim 3 \%$ of all cancers in the United States, with a very low survival rate $(<5 \%)$ [17]. miR-24 is overexpressed in pancreatic cancer. Luciferase assays have shown that Bim (also known as BCL2L11) is direct target of miR-24. BCL2L11 belongs to the BCL2 family of proapoptotic genes, however its biological function is not fully established [18].

An increase in miR-24 and decrease in BCL2L11 expression have been demonstrated in pancreatic cancer cells, resulting in cell proliferation and reduced apoptosis [18]. It has been proposed that cell growth, apoptosis and ring formation of vascular endothelial cells are regulated by miR-24 and BCL2L11. Animal models have been used to demonstrate that miR-24 promotes the growth of pancreatic tumors, and that BCL2L11 inhibits tumor growth. A study infected pancreatic cancer cells with lentivirus particles to overexpress miR-24 or BCL2L11, and the cells were then implanted into immunodeficient mice. Tumor size was increased in mice implanted with cells that had high expression of miR-24, and tumor growth was inhibited by overexpression of BCL2L11. These tumor implantation experiments in mice provide strong evidence that miR-24 and BCL2L11 regulate tumor growth in pancreatic cancer [18].

\section{miR-24 and Lung Cancer}

Each year, $\sim 1.61$ million new cases of lung cancer are diagnosed, with $\sim 1.38$ million deaths. Non-small cell lung cancer in the most common lung cancer subtype, accounting for $\sim 85 \%$ of cases [19].

Little is known about the role of miR-24 in non-small cell lung cancer. A study by Le et al. identified that miR-24 expression was higher in the serum of patients with lung cancer patients than in healthy controls [7]. Zhao et al. reported that miR-24 was overexpressed in serum samples and tissue samples from patients with non-small cell lung cancer [20]. Additionally, overexpression of miR-24 in serum was associated with shorter survival in patients with non-small cell lung cancer. This study also analyzed the mechanisms by which miR-24 may participate in the development of non-small cell lung cancer, and it was determined that miR-24 acts as oncomiR by regulating the expression of Nuclear Apoptosis Inducing Factor 1 (NAIF1), which results in increased cell proliferation; thus, miR-24 may be a useful a biomarker for the prognosis of non-small cell lung cancer [20].

Yan et al. analyzed the expression of miR-24 and sex-determining region Y-box 7 (SOX7) in lung cancer cell lines [21]. Cell proliferation was analyzed in A549 cells transfected with miR-24 mimics, which increased in cell proliferation, and the opposite effect was observed in A549 cells transfected with miR-24 inhibitors, suggesting that miR-24 increases the proliferation of lung cancer cells [21]. Luciferase assays confirmed that SOX7 is direct target of miR-24-3p. Additionally, miR-24 was shown to increase xenograft tumor growth in mice by targeting SOX7. These results provide evidence that miR-24 may have an oncomiR function in lung cancer by targeting SOX7 [21].

\section{miR-24 and Leukemia}

Acute leukemia is the most common childhood cancer. Previous studies have identified the processes by which miR- 24 is involved in hematopoiesis and the differentiation of hematopoietic cell lines; however, few studies have investigated the role of miR-24 in acute leukemia. Organista et al. analyzed the expression of miR-24 in patients with acute leukemia [9], noting that patients with acute leukemia had increased expression of miR-24 compared to healthy subjects. Furthermore, the expression levels of miR-24 were higher in patients with acute myeloid leukemia than in patients with acute lymphoid leukemia.

It has also been reported that there is a significant correlation between miR-24 expression levels and the risk of relapse in patients with acute leukemia. Patients with high expression of miR-24 have a significantly higher risk of relapse compared to patients with low expression of miR-24. Furthermore, acute leukemia patients with high expression of miR-24 tend to have shorter survival compared to patients with low expression of miR-24 [9]. Thus, high expression of miR-24 is associated with poor prognosis and short survival in patients with acute leukemia. miR-24 may be an independent prognostic marker that is able to determine the clinical behavior of patients with acute leukemia [9].

\section{Conclusion}

Dysregulation of miRNAs expression has been reported to be associated with carcinogenesis, invasion and metastasis. miRNAs can be detected and measured in serum, plasma and tissue from patients with cancer, and thus be used as biomarkers; however, more research is required so that they can be used clinically. Previous studies have revealed associations between the levels of expression of various miRNAs and the prognosis of cancer patients.

miR-24 is an miRNA that is considered to be an oncomiR as it is overexpressed in different types of cancer, such as gastric cancer, where it decreases the expression of SOCS6 resulting in increased cell proliferation and decreased apoptosis. In breast cancer, the overexpression of miR-24 is associated with the development of metastasis and the risk of relapse. It is also associated with the regulation of genes such as ING5 that control the cell cycle, proliferation and apoptosis in this type of cancer. In pancreatic cancer, the overexpression of miR-24 leads to the decrease in BCL2L11 expression, and in lung cancer, miR-24 decreases NAIF1 and SOX7 expression, which alters cell proliferation and apoptosis. Finally, in leukemia overexpression of miR-24 is associated with poor patient prognosis and reduced survival duration.

All the findings discussed above indicate that miR-24 regulates the expression of genes involved in key processes of carcinogenesis, such as cell proliferation and apoptosis. The overexpression of miR-24 may be a predictor of poor prognosis and, together with the measurement of other established markers for each type of cancer, could be used to help improve the survival and prognosis of patients with cancer. 
Citation: Rivas-Alarcón A, Organista-Nava J, Illades-Aguiar B, Leyva-Vázquez MA, Gómez-Gómez Y. miR-24 and its Role as an Oncogene in Cancer. J Hum Anat Physiol 2019;3(1): 4.

\section{Perspectives}

A clear understanding of the mechanisms by which miR-24 acts an oncogene could allow miR-24 to be used as a prognostic marker; however, further studies are required corroborate this.

More basic and clinical research is required to fully understand the regulation and role of miR-24 in cancer. With a clear understanding of the molecular mechanisms that regulate miR-24, this miRNA could potentially be used as a therapeutic target for different types of cancer, as it has been established that miR-24 is involved in the regulation of genes that mediate key signaling pathways during the development of metastasis. The measurement of miR-24 expression levels could help to identify patients at high risk of relapse and those with poor prognosis.

\section{References}

1. Bentwich I, Avniel A, Karov Y, Ahoronov Y, Gilad S, et al. (2005) Identification of hundreds of conserved and nonconserved human microRNAs. Nat Genet 37: 766-776.

2. Macfarlane L, Murphy $P(2010)$ MicroRNA: biogenesis, function and role in cancer. Curr Genomics 11: 537-561.

3. Gulyaeva L, Kushlinskiy N (2016) Regulatory mechanisms of microRNA expression. J Transl Med 14: 143

4. Lee Y, Dutta A (2009) MicroRNAs in cancer. Annu Rev Pathol 4: 199-227.

5. Zhu K, Liu D, Lai H, Li J, Wang C (2016) Developing miRNA therapeutics for cardiac repair in ischemic heart disease. J Thorac Dis 8: E918-E927.

6. Cui S, Liao X, Ye C, Yin X, Liu M, et al. (2017) ING5 suppresses breast cancer progression and is regulated by miR-24. Mol Cancer 16: 89.

7. Le H, Zhu W, Chen D, He J, Huang Y, et al. (2012) Evaluation of dynamic change of serum miR-21 and miR-24 in pre- and post-operative lung carcinoma patients. Med Oncol 29: 3190-3197.

8. Zhang H, Duan J, Qu Y, Deng T Liu R, et al. (2016) Onco-miR-24 regulates cell growth and apoptosis by targeting BCL2L11 in gastric cancer. Protein Cell 7: 141-151.
9. Organista-Nava J, Gómez-Gómez Y, Illades-Aguiar B, Del Carmen AlarcónRomero L, Saavedra-Herrera MV, et al. (2015) High miR-24 expression is associated with risk of relapse and poor survival in acute leukemia. Oncol Rep 33: 1639-1649.

10. Quan J, Liu S, Dai K, Jin L, He T, et al. (2017) MicroRNA-23a/24-2/27a as a potential diagnostic biomarker for cancer: A systematic review and metaanalysis. Mol Clin Oncol 8: 159-169.

11. Hua K, Chen Y, Tang Y, Huang T, Lin Y, et al. (2017) MicroRNA-23a/27a/24-2 cluster promotes gastric cancer cell proliferation synergistically. Oncol Lett 16: $2319-2325$

12. Khodadadi-Jamayran A, Akgol-Oksuz B, Afanasyeva Y, Heguy A, Thompson $M$, et al. (2018) Prognostic role of elevated mir-24-3p in breast cancer and its association with the metastatic process. Oncotarget 9: 12868-12878.

13. Basova P, Pesta M, Sochur M, Stopka M (2017) Prediction Potential of Serum miR-155 and miR-24 for Relapsing Early Breast Cancer. Int J Mol Sci 18: pii: E2116.

14. Roscigno G, Puoti I, Giordano I, Donnarumma E, Russo V, et al. (2017) MiR-24 induces chemotherapy resistance and hypoxic advantage in breast cancer. Oncotarget 8: 19507-19521.

15. Gunduz M, Gunduz Z, Rivera R, Nagatzuka H (2008) The Inhibitor of Growth (ING) gene family: potential role in cancer therapy. Curr Cancer Drug Targets 8: $275-284$

16. Gou WF, Shen DF, Yang XF, Zhao S, Liu YP, et al. (2015) ING5 suppresses proliferation, apoptosis, migration and invasion, and induces autophagy and differentiation of gastric cancer cells: a good marker for carcinogenesis and subsequent progression. Oncotarget 6: 19552-19579.

17. Siegel R, Ma J, Zou Z, Jemal A (2014) Cancer statistics, 2014. CA Cancer J Clin 64: 9-29.

18. Liu R, Zhang H, Wang X, Zhou L, Li H, et al. (2015) The miR-24-Bim pathway promotes tumor growth and angiogenesis in pancreatic carcinoma. Oncotarget 6: 43831-43842.

19. Jemal A, Bray F, Center MM, Ferlay J, Ward E, et al. (2011) Global cancer statistics. CA Cancer J Clin 61: 69-90

20. Zhao G, Liu L, Jin S, Jiang S, Cao S, et al. (2015) Upregulation of miR-24 promotes cell proliferation by targeting NAIF1 in non-small cell lung cancer. Tumor Biol 36: 3693-3701.

21. Yan L, Ma J, Zhu Y, Zan J, Wang Z, et al. (2016) MiR-24-3p promotes cell migration and proliferation in lung cancer by targeting SOX7. J Cell Biochem 119: 3989-3998. 